\title{
Chemical abundances of the PRGs UGC 7576 and UGC 9796
}

\section{Testing the formation scenario}

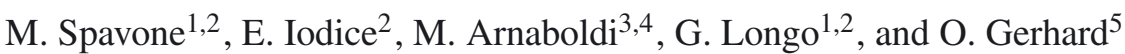 \\ ${ }^{1}$ Dipartimento di Scienze Fisiche, Universitá Federico II, via Cinthia 6, 80126 Napoli, Italy \\ e-mail: spavone@na.infn.it \\ 2 INAF - Astronomical Observatory of Naples, via Moiariello 16, 80131 Napoli, Italy \\ 3 European Southern Observatory, Karl-Schwarzschild-Strae 2, 85748 Garching bei München, Germany \\ ${ }^{4}$ INAF, Osservatorio Astronomico di Pino Torinese, 10025 Pino Torinese, Italy \\ 5 Max-Plank-Institut für Extraterrestrische Physik, Giessenbachstrae, 85741 Garching bei München, Germany
}

Received 26 January 2011 / Accepted 7 April 2011

\begin{abstract}
Context. The study of both the chemical abundances of HII regions in polar ring galaxies and their implications for the evolutionary scenario of these systems has been a step forward both in tracing the formation history of the galaxy and giving hints toward the mechanisms at work during the building of a disk by cold accretion process. It is now important to establish whether such results are typical of the class of polar disk galaxies as a whole.

Aims. The present work aims at checking the cold accretion of gas through a "cosmic filament" as a possible scenario for the formation of the polar structures in UGC 7576 and UGC 9796. If these form by cold accretion, we expect the HII regions abundances and metallicities to be lower than those of same-luminosity spiral disks, with values of $Z \sim 1 / 10 Z_{\odot}$, as predicted by cosmological simulations.

Methods. We used deep long-slit spectra, obtained with DOLORES@TNG in the optical wavelengths, of the brightest HII regions associated with the polar structures to derive their chemical abundances and star formation rate. We used the empirical methods, based on the intensities of easily observable lines, to derive the oxygen abundance $12+\log (\mathrm{O} / \mathrm{H})$ of both galaxies. Such values are compared with those typical of different morphological galaxy types of comparable luminosity.

Results. The average metallicity values for UGC 7576 and UGC 9796 are $Z=0.4 Z_{\odot}$ and $Z=0.1 Z_{\odot}$, respectively. Both values are lower than those measured for ordinary spirals of similar luminosity, and UGC 7576 presents no metallicity gradient along the polar structure. These data, together with other observed features available for the two PRGs in previous works, are compared with the predictions of simulations of tidal accretion, cold accretion, and merging to disentangle these scenarios.
\end{abstract}

Key words. galaxies: abundances - galaxies: evolution - galaxies: formation - galaxies: individual: UGC 7576 galaxies: individual: UGC 9796 - galaxies: peculiar

\section{Introduction}

How galaxies acquire their gas is an open issue in the models of galaxy formation. Recent theoretical works, which are supported by many numerical simulations, have argued that cold accretion plays a major role (Katz \& White 1993; Katz et al. 1994; Kereš et al. 2005; Dekel \& Birnboim 2006; Dekel \& Birnboim 2008; Bournaud \& Elmegreen 2009). Kereš et al. (2005) studied the physics of the cold mode of gas accretion in detail and they find that it is generally directed along filaments, allowing galaxies to draw gas from large distances. In particular, the cold accretion is a key mechanism for providing gas to disk galaxies (Brooks et al. 2009).

Recent simulations of disk formation in a cosmological context performed by Agertz et al. (2009) revealed that the so-called chain galaxies and clump clusters, found only at higher redshifts (Elmegreen et al. 2007), are a natural outcome both of early epoch enhanced gas accretion from cold dense streams and of tidally and ram-pressured stripped material from minor mergers and satellites. This freshly accreted cold gas settles into large disk-like systems. This scenario reproduces the observed morphology and global rotation of disks and predicts both a realistic metallicity gradient and a star formation rate $(S F R)$ of $20 M_{\odot} / \mathrm{yr}$.
Agertz et al. (2009) find solar metallicity for the inner disk, while in the clump forming region it is only $\sim 1 / 10 Z_{\odot}$ owing to the accretion of pristine gas in the cold streams mixed with stripped satellite gas.

Simulations also show that the interaction region between the newly formed disk and the cold streams can also cause it to be misaligned with the initial galactic disk. Based on very limited statistics, Agertz et al. (2009) suggest that this misalignment might not be typical, and that it comes from a third cold stream that is perpendicular to the main filament. More recent analysis shows that the accretion of gas along misaligned filaments with respect to the disk plane are more common, and it leaves traces down to low redshift (Dekel et al. 2009b; Roškar et al. 2010). An almost polar ring can result even as an extreme case of this process and, as suggested by Agertz et al. (2009), it could be responsible for forming polar disks.

Hydrodynamical simulations performed by Macciò et al. (2006) and Brook et al. (2008) have shown that the formation of a polar disk galaxy can occur naturally in a hierarchical universe, where most low-mass galaxies are assembled through the accretion of cold gas infalling along filamentary structures. According to Macciò et al. (2006), the polar disk forms from cold gas that flows along the extended $\sim 1 \mathrm{Mpc}$ filament into the virialized 
dark matter halo. The gas streams into the center of the halo on an orbit that is offset from radial infall. As it reaches the center, it impacts with gas in the halo of the host galaxy and with the warm gas flowing along the opposite filament. Only the gas accreted perpendicular to the major axis of the potential can survive for more than a few dynamical lifetimes.

Brook et al. (2008) argue that polar disk galaxies are extreme examples of the misalignment of angular momentum that occurs during the hierarchical structure formation: an inner disk starts forming shortly after the last major merger at $z \sim 2$. Because of its gas rich nature, the galaxy rapidly forms a new disk whose angular momentum is determined by the merger orbital parameters. Later, gas continues to be accreted but in a plane that is almost perpendicular to the inner disk. At $z \sim 0.8$ the central galaxy is still forming stars in a disk, while the bulk of new star formation is in a highly inclined polar disk. The inner disk has exhausted its gas by $z \sim 0.5$, while gas continues to fall onto the polar disk. From this point on, star formation occurs exclusively in the polar disk, which remains stable for at least 3 Gyr. The formation mechanisms described above can self-consistently explain both the morphology and kinematics of a polar disk galaxy. In particular, the predictions turn out to be consistent with many features (e.g. colors and color gradients, longevity, spiral arms, HI content, and distribution) observed for the polar disk galaxy NGC 4650A.

NGC 4650A is the prototype of the wide polar ring galaxies (PRGs), and it has been studied in detail to understand its formation and physical properties (Arnaboldi et al. 1997; Gallagher et al. 2002; Iodice et al. 2002a; Swaters \& Rubin 2003; Bournaud \& Combes 2003; Iodice et al. 2006) and to constrain many of the processes at work during galaxy interactions and merging. Very recently, Spavone et al. (2010) have used deep longslit spectroscopy with FORS2@ESO-VLT to study the abundance ratios and metallicities of the HII regions associated to the polar disk in NGC 4650A and to test the cold accretion scenario for this object. The chemical abundance is one of the key parameters to be estimated in a galaxy disk and directly compared with the theoretical predictions; in fact, if the gas is essentially primordial, it should have a very low abundance of the heavy elements.

The main results obtained for NGC 4650A show i) that it has lower metallicity than spiral galaxy disks of the same total luminosity, where $Z=0.2 Z_{\odot}$, which is consistent with values predicted for the formation of disks by cold accretion processes (Agertz et al. 2009); ii) there is no metallicity gradient along the polar disk, which suggests that the metal enrichment is not influenced by the stellar evolution of the older central spheroid, so that the disk was formed later by the infall of metal-poor gas from outside that is still forming the disk. The latter is also a characteristic of some other PRGs (Brosch et al. 2010) and of LSB galaxies (de Blok \& van der Hulst 1998), which have colors, metallicities, age, and brightness similar to those of PRGs suggesting that the infall of metal-poor gas may reasonably fit all observational evidence.

Spavone et al. (2010) have led the way in implementing a test for the cold accretion. This study has revealed an indirect and well-based check for the cold accretion scenario of disk formation. In this work, we would like to perform the same kind of analysis on the two PRGs, UGC 7576 and UGC 9796. These two objects are similar to NGC 4650A, and such a morphological similarity may suggest a similar formation mechanism. The polar structures are more extended than the central spheroids, therefore, like NGC 4650A, they were classified as wide PRG (Whitmore et al. 1990). They are characterized by exponential

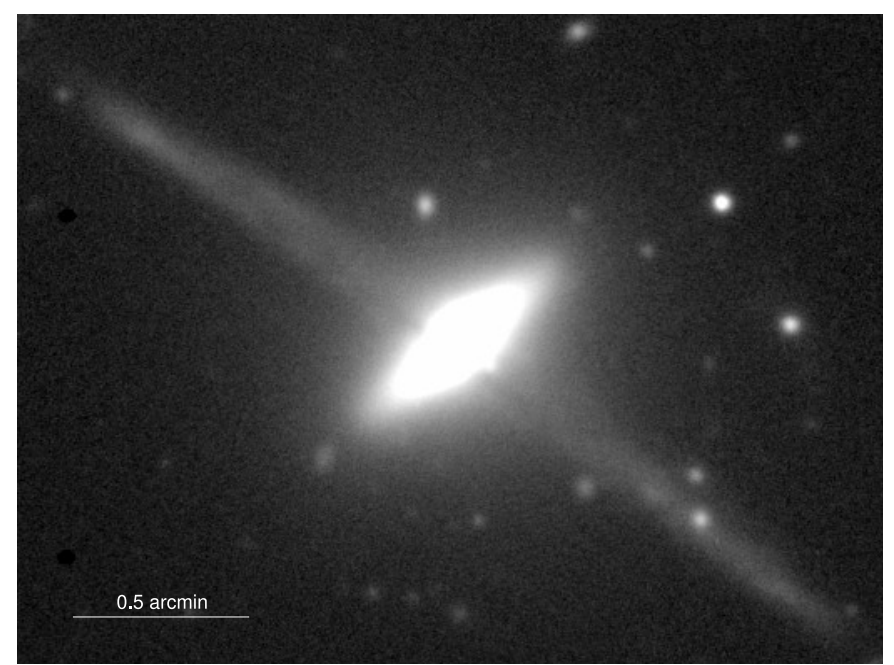

Fig. 1. $R$-band image of the polar ring galaxy UGC 7576.

Table 1. Global properties of UGC 7576 and UGC 9796 compared to those observed for NGC 4650A.

\begin{tabular}{lccc}
\hline \hline Parameter & UGC 7576 & UGC 9796 & NGC 4650A \\
\hline RA (J2000) & $12^{\mathrm{h}} 27^{\mathrm{m}} 41.8^{\mathrm{s}}$ & $15^{\mathrm{h}} 15^{\mathrm{m}} 56.3^{\mathrm{s}}$ & $12^{\mathrm{h}} 44^{\mathrm{m}} 49.0^{s}$ \\
Dec (J2000) & $+28^{\mathrm{d}} 41^{\mathrm{m}} 53^{\mathrm{s}}$ & $+43^{\mathrm{d}} 10^{\mathrm{m}} 00^{\mathrm{s}}$ & $-40^{d} 42^{\mathrm{m}} 52^{s}$ \\
Helio. radial velocity & $7022 \mathrm{~km} \mathrm{~s}^{-1}$ & $5406 \mathrm{~km} \mathrm{~s}^{-1}$ & $2880 \mathrm{~km} \mathrm{~s}^{-1}$ \\
Redshift & 0.02342 & 0.01832 & 0.009607 \\
Distance & $94 \mathrm{Mpc}$ & $72 \mathrm{Mpc}$ & $38 \mathrm{Mpc}$ \\
Central galaxy & & & \\
$M_{B}$ & -19.15 & -17.93 & -18.83 \\
$B-V$ & +0.84 & +0.92 & +0.78 \\
$V-R$ & +0.46 & +0.55 & \\
Polar structure & & & \\
$M_{B}$ & -17.5 & -17.0 & -17.0 \\
$M(\mathrm{HI})\left(M_{\odot}\right)$ & $2.7 \times 10^{9}$ & $2.6 \times 10^{9}$ & $8.0 \times 10^{9 a}$ \\
$M(\mathrm{HI}) / L_{B}$ & 0.6 & 1.5 & 4 \\
$B-V$ & $+0.70^{b}$ & $+0.57^{b}$ & $+0.26^{c}$ \\
$\mu_{B}$ & 24.3 & 24.5 & 22.6 \\
$R_{25}$ & 13.6 & 10.8 & 7.0 \\
$r_{\max }$ & $40^{\prime \prime}$ & $60^{\prime \prime}$ & $40^{\prime \prime}$ \\
\hline
\end{tabular}

References. ${ }^{(a)}$ Arnaboldi et al. (1997); ${ }^{(b)}$ Reshetnikov et al. (1994); ${ }^{(c)}$ Iodice et al. (2002).

surface brightness profiles, very blue colors, knotty appearance, and a large amount of HI gas, all associated to these components. The most relevant feature in UGC 9796 is that the HI distribution resembles that of a disk in differential rotation rather than a ring (Reshetnikov et al. 1994; Cox et al. 2006), like in NGC 4650A (Arnaboldi et al. 1997). Given the new results for NGC 4650A, it is now important to establish whether the lack of metallicity gradient and low metallicity and the inferred accretion of lowmetallicity material after the formation of the central spheroid, are typical for the class of wide PRGs as a whole. To this aim we have studied the PRGs UGC 7576 and UGC 9796, that are suitable systems to investigate this question further.

\section{Properties of the PRGs UGC 7576 and UGC 9796}

\subsection{UGC 7576}

UGC 7576 (Fig. 1) is a kinematically confirmed PRG (Whitmore et al. 1990) and its main properties are listed in Table 1. 
This object is at a distance of about $94 \mathrm{Mpc}$, based on $H_{0}=75 \mathrm{~km} \mathrm{~s}^{-1} \mathrm{Mpc}^{-1}$ and has a heliocentric radial velocity of $V=7022 \mathrm{~km} \mathrm{~s}^{-1}$, which implies that 1 arcsec $=0.45 \mathrm{kpc}$. The kinematics show that the polar structure of UGC 7576 is more like a ring than a disk, given that the rotation curve does not show any differential rotation, unlike those of NGC 4650A and UGC 9796. Reshetnikov et al. (1994) studied the global morphology of UGC 7576 and were able to distinguish three components: $i$ ) the main central body with elliptical isophotes; $i i$ ) a narrow polar ring crossing the central region of the main galaxy; and iii) a faint outer envelope. The gradient of the surface brightness distribution of the central galaxy decreases at $r \geq 20^{\prime \prime}$, while both $B-V$ and $V-R$ color indices decrease slightly with radius.

The distribution of color indices is somewhat asymmetric, suggesting that the polar ring is projected not exactly on the nucleus but with a small NW displacement. The color asymmetry correlates with the asymmetry in the hydrogen distribution (Schechter et al. 1984). Moreover, the plane of the disk reveals a warping, which suggests that the ring has not yet settled into the equilibrium plane.

Surface brightness distribution along the major axis of the ring is very symmetric, and the surface brightness is approximately constant $\left(\mu_{B} \sim 24.3\right)$ up to $r \sim 35^{\prime \prime}$, then falls abruptly. Also the colors show no evident changes within the region of constant brightness.

UGC 7576 is also embedded in a faint outer envelope, whose major axis position angle coincides with the position angle of the polar ring, and this suggests a common origin for both components (Reshetnikov et al. 1994).

Reshetnikov \& Combes (1994) have analyzed the $\mathrm{H}_{\alpha}$ spectroscopy for a sample of PRGs, including both UGC 7576 and UGC 9796. In the case of UGC 7576, the $\mathrm{H}_{\alpha}$ rotation curve along the polar structure was consistent with the HI rotation curve obtained by Schechter et al. (1984), and both curves are straight lines with the same gradient. This led the authors to conclude that the ring is more likely an edge-on narrow annulus. By assuming a spherical mass distribution, Reshetnikov \& Combes (1994) estimated a total mass-to-light ratio of $M / L_{B} \sim$ $11.3 M_{\odot} / L_{\odot}$ within the last measured point of the rotation curve (where the velocity reaches its maximum value). This value, together with the $M / L_{B}$ for UGC 9796, is among the highest $M / L_{B}$ estimated for the whole sample of PRGs. Reshetnikov \& Combes (1994) also performed a simple dynamical mass model for both the PRGs studied in this work. $\mathrm{n}$ the hypothesis of a spherical dark halo, for UGC 7576 they find a dark-to-luminous mass ratio of about 1.3 , inside the polar ring radius.

\subsection{UGC 9796}

UGC 9796 (Fig. 2), also known as IIZw 73, is at a distance of about $72 \mathrm{Mpc}$ based on $H_{0}=75 \mathrm{~km} \mathrm{~s}^{-1} \mathrm{Mpc}^{-1}$ and heliocentric radial velocity of $V=5406 \mathrm{~km} \mathrm{~s}^{-1}$, which implies that 1 arc$\mathrm{sec}=0.35 \mathrm{kpc}$. It has one of the most non polar PRG. Its apparent major axis is in fact only $65^{\circ}$ from the major axis of the central S0, rotationally supported galaxy, and this implies a rapid rate of differential precession. The polar structure is less symmetric than in UGC 7576, and the distribution of the colors is also asymmetric, with the NE side considerably redder than the SW side. The color asymmetry coincides with the asymmetry of the HI density distribution. The HI gas is all associated with the polar structure, which thus contains as many baryons as the host galaxy (Schechter et al. 1984), and shows a central hole at about $25^{\prime \prime}$. The huge mass-to-light ratio of $M_{\mathrm{dyn}} / L_{B} \simeq 50$ in solar units has lead Cox et al. (2006) to conclude that most of the mass in

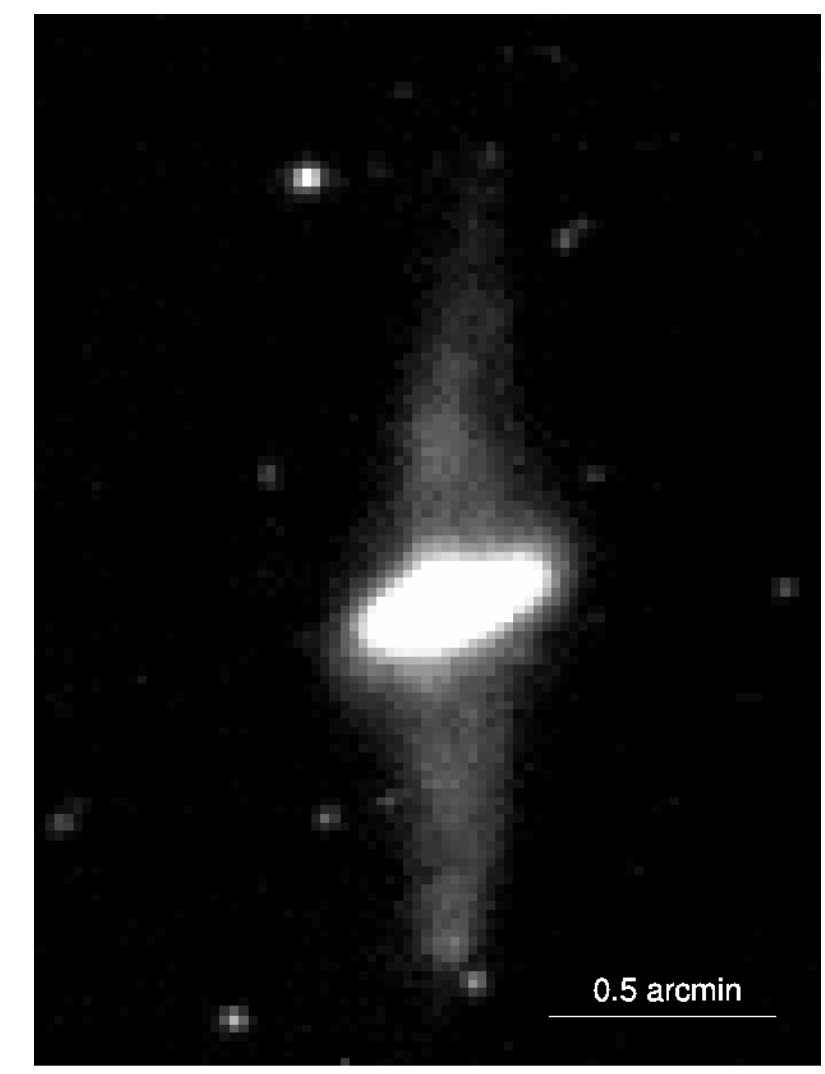

Fig. 2. Optical image of the polar ring galaxy UGC 9796, obtained by the authors at TNG telescope.

this system is dark. As in the case of UGC 7576, the $\mathrm{H}_{\alpha}$ rotation curve (Reshetnikov \& Combes 1994) is in good agreement with the HI rotation curve (Schechter et al. 1984). The shape of the rotation curve indicates that this component is actually a differentially rotating disk, rather than a ring, very similar to the polar disk in NGC 4650A.

In Table 1 we summarize the photometric and HI observed quantities for UGC 7576 and UGC 9796 and add those of NGC 4650A for reference in the same table.

\section{Observation and data reduction}

The spectra analyzed in this work were obtained with DOLORES@TNG (Device Optimized for the LOw RESolution), in visitor mode during the observing run A21TAC54 (on May 2010). DOLORES is installed at the Nasmyth B focus of the TNG and is equipped with the E2V 4240 CCD with an angular resolution of $0^{\prime} .252 \mathrm{pix}^{-1}$.

The adopted slit was $2^{\prime \prime}$ wide and it was aligned along both the north and south sides of the polar structures of the two galaxies UGC 7576 and UGC 9796, at PA $=53^{\circ}$ and PA $=16^{\circ}$, respectively (see Fig. 3), in order to include the most luminous HII regions in the polar structures. The total integration time for each object is $4 \mathrm{~h}$, with an average seeing of 1 '. 2 .

At the systemic velocities of UGC 7576 and UGC 9796, to cover the red-shifted emission lines of [OII] $\lambda 3727, \mathrm{H}_{\gamma}(\lambda 4340)$, [OIII] $\lambda 4363,[\mathrm{OIII}] \lambda \lambda 4959,5007, \mathrm{H}_{\beta}(\lambda 4861)$ and $\mathrm{H}_{\alpha}(\lambda 6563)$, the grism LR-B was used in the 3600-6800 A wavelength range, with a dispersion of $2.52 \AA /$ pix. 

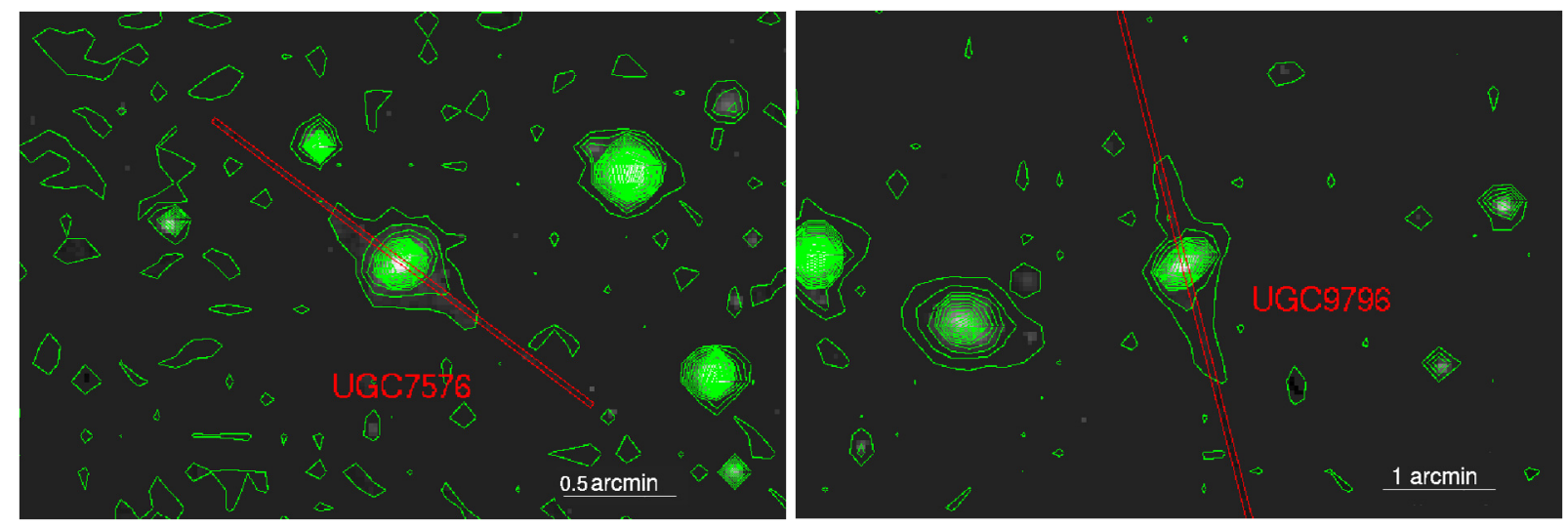

Fig. 3. Optical image of UGC 7576 (left) and UGC 9796 (right), with superimposed slits used to acquire data analyzed in this work.

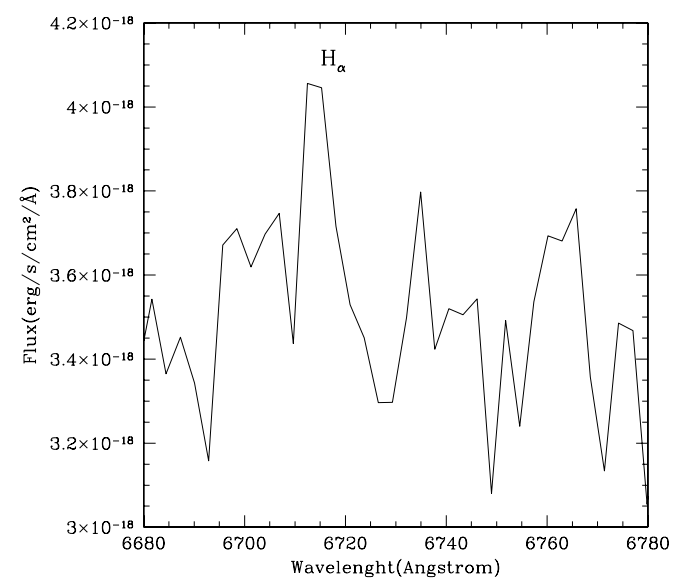

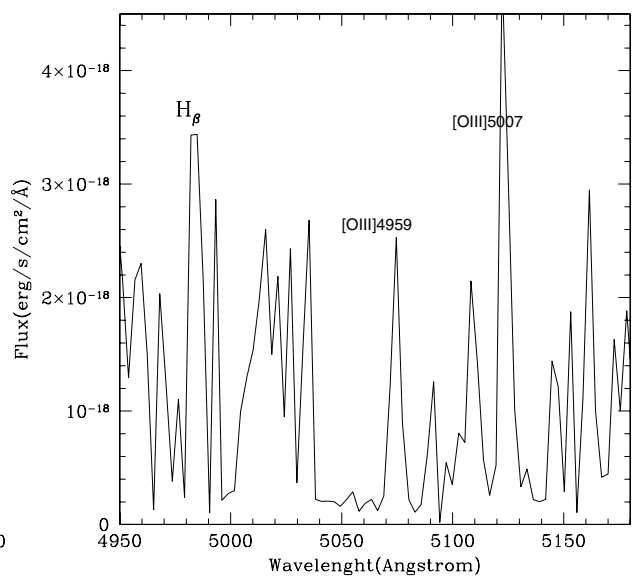

Fig. 4. Spectrum of UGC 7576 obtained by summing up $1 \mathrm{D}$ spectra extracted along both the north and south sides of the polar structure.
The data reduction was carried out using the CCDRED package in the $\operatorname{IRAF}^{1}$ (Image Reduction and Analysis Facility) environment. The main strategy adopted for each dataset included dark subtraction ${ }^{2}$, flat-fielding correction, sky subtraction, and rejection of bad pixels. Wavelength calibration was achieved by means of comparison spectra of $\mathrm{Hg}+\mathrm{Ne}$ lamps acquired for each observing night, using the IRAF TWODSPEC.LONGSLIT package. The sky spectrum was extracted at the outer edges of the slit, for $r \geq 30$ arcsec from the galaxy center, where the surface brightness is fainter than $24 \mathrm{mag} / \operatorname{arcsec}^{2}$, and subtracted off each row of the two dimensional spectra by using the IRAF task BACKGROUND in the TWODSPEC.LONGSLIT package. On average, a sky subtraction better than $1 \%$ was achieved. The sky-subtracted frames were co-added to a final median-averaged 2D spectrum.

The final step of the data processing is the flux calibration of each 2D spectrum, by using observations of the standard star Feige66 and the standard tasks in IRAF (STANDARD, SENSFUNC and CALIBRATE). To perform the flux calibration, we extracted a 1-D spectrum of the standard star to find the calibration function, and then we extracted a set of 1-D spectra of the galaxy by summing up a number of lines corresponding to the slit width. Since the slit width was $2^{\prime \prime}$ and the scale of the instrument was $0.252^{\prime \prime} /$ pix, we collapsed eight lines to obtain each

\footnotetext{
1 IRAF is distributed by the National Optical Astronomy Observatories, which is operated by the Associated Universities for Research in Astronomy, Inc. under cooperative agreement with the National Science Foundation.

${ }^{2}$ Bias frame is included in the dark frame.
}

1-D spectrum. Finally we applied the flux calibration to this collection of spectra. The wavelength and flux-calibrated spectra are shown in Figs. 4 and 5.

The fluxes of these emission lines were measured using the IRAF SPLOT routine, which provides an interactive facility to display and analyze spectra. We evaluated the flux and equivalent width by marking two continuum points around the line to be measured. The linear continuum is subtracted and the flux is determined by simply integrating the line intensity over the local fitted continuum. The errors on these quantities are calculated, following Pérez-Montero \& Díaz (2003), by the relation $\sigma_{1}=\sigma_{\mathrm{c}} N^{1 / 2}[1+E W /(N \Delta)]^{1 / 2}$, where $\sigma_{1}$ is the error in the line flux, $\sigma_{\mathrm{c}}$ the standard deviation in a box near the measured line and represents the error in the continuum definition, $N$ is the number of pixels used to measure the flux, $E W$ the equivalent width of the line, and $\Delta$ the wavelength dispersion in $\AA /$ pixel.

\subsection{Reddening correction}

Reduced and flux-calibrated spectra and the measured emission line intensities were corrected for the reddening, which account both for that intrinsic to the source and to the Milky Way. By comparing the intrinsic Balmer decrement $\mathrm{H}_{\alpha} / \mathrm{H}_{\beta}=2.89$, we derived the visual extinction $A(V)$ and the color excess $E(B-V)$ by adopting the mean extinction curve by Cardelli et al. (1989) $A(\lambda) / A(V)=a(x)+b(x) R_{V}$, where $R_{V}[\equiv A(V) / E(B-V)]=3.1$ and $x=1 / \lambda$. All the emission lines in our spectra are in the $o p$ tical/NIR range (see Cardelli et al. 1989), so we used the average 

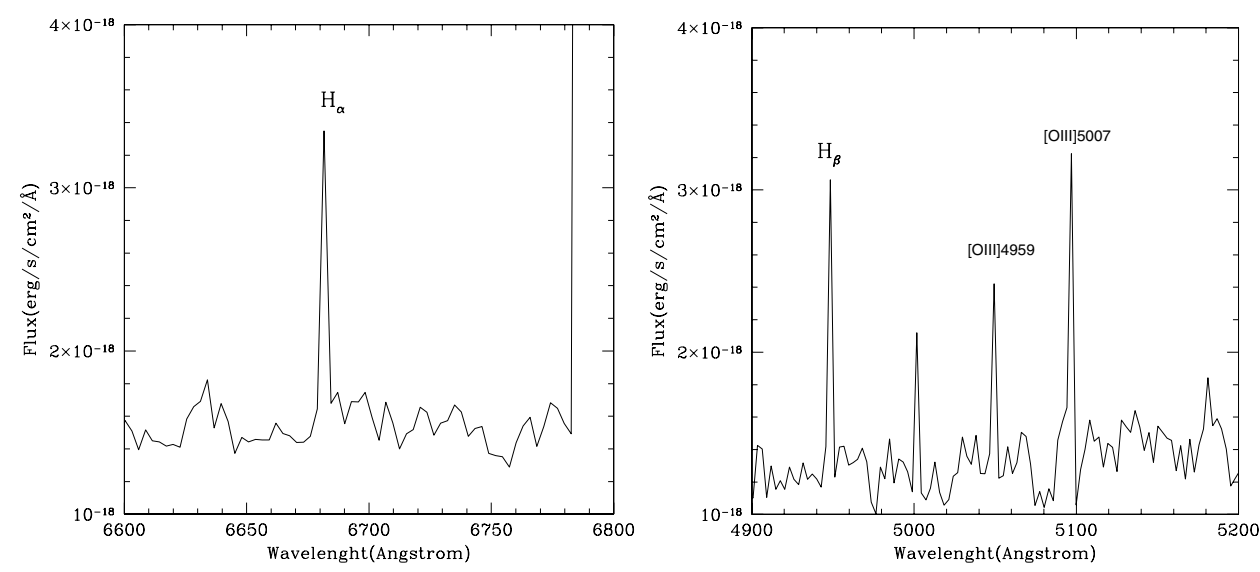

Fig. 5. Spectrum of UGC 9796 obtained by summing up $1 \mathrm{D}$ spectra extracted along both the north and south sides of the polar structure.
$R_{V}$-dependent extinction law derived for these intervals to perform the reddening correction.

We derived the average observed Balmer decrements for both galaxies, which are

$$
\begin{aligned}
& \left(\mathrm{H}_{\alpha} / \mathrm{H}_{\beta}\right)_{\text {UGC } 7576}=3.18 \pm 2.10 \\
& \left(\mathrm{H}_{\alpha} / \mathrm{H}_{\beta}\right)_{\text {UGC } 9796}=2.24 \pm 1.30,
\end{aligned}
$$

while the color excess obtained by using these observed decrements are

$$
\begin{aligned}
& {[E(B-V)]_{\mathrm{UGC}} 7576=0.09 \pm 0.36} \\
& {[E(B-V)]_{\mathrm{UGC}} 9796=-0.25 \pm 0.92 .}
\end{aligned}
$$

The negative value of the color excess for UGC 9796 indicates the presence of stars that are bluer and hotter than normal, and thus they have $V$ apparent magnitude greater than the $B$ one, leading to a negative $B-V$ color. In general, stars hotter than Vega, which have $E(B-V)=0$, have negative color excess (Masana et al. 1998).

Such values of $E(B-V)$ are used to derive the extinction $A_{\lambda}$ through the Cardelli's law. Finally, the corrected fluxes are given by

$$
\frac{F_{\text {int }}^{\lambda}}{F_{\text {int }}^{\mathrm{H}_{\beta}}}=\frac{F_{\text {obs }}^{\lambda}}{F_{\text {obs }}^{\mathrm{H}_{\beta}}} 10^{0.4\left[A_{\lambda}-A_{\mathrm{H}_{\beta}}\right]} .
$$

\section{Empirical oxygen abundances determination}

The analysis of nebular spectra in HII regions is the best tool for determining chemical abundances in spiral and irregular galaxies. The abundances of several elements can be determined by using strong emission lines that are clearly visible in the spectra.

The main aim of this work is to measure the Oxygen abundance parameter $R_{23}=([\mathrm{OII}] \lambda 3727+[\mathrm{OIII}] \lambda \lambda 4959+5007) / \mathrm{H}_{\beta}$ (Pagel et al. 1979), and consequently the oxygen abundance $12+\log (\mathrm{O} / \mathrm{H})$ and the metallicity of the HII regions in the polar disks of UGC 7576 and UGC 9796, following the procedure outlined by Spavone et al. (2010) for NGC 4650A.

The Empirical methods are based on the cooling properties of ionized nebulae, which translate into a relation between emission-line intensities and oxygen abundance. Several abundance calibrators have been proposed based on different emission-line ratios: $R_{23}$ (Pagel et al. 1979) and $S_{23}$ (Díaz \& Pérez-Montero 2000). Among the others, in this work we used the so-called P-method introduced by Pilyugin (2001).
Pilyugin (2001) realized that, for fixed oxygen abundances, the value of $X_{23}=\log R_{23}$ varies with the excitation parameter $P=R_{3} / R_{23}$, where $R_{3}=\mathrm{OIII}[4959+5007] / \mathrm{H}_{\beta}$, and proposes that this latter parameter could be used in the oxygen abundance determination. This method, called the "P-method", proposes using a more general relation of the type $\mathrm{O} / \mathrm{H}=f\left(P, R_{23}\right)$, than the relation $\mathrm{O} / \mathrm{H}=f\left(R_{23}\right)$ used in the $R_{23}$ method. The equation related to this method is

$$
12+\log (\mathrm{O} / \mathrm{H})_{P}=\frac{R_{23}+54.2+59.45 P+7.31 P^{2}}{6.07+6.71 P+0.371 P^{2}+0.243 R_{23}}
$$

where $P=R_{3} / R_{23}$. It can be used for oxygen abundance determination in moderately high-metallicity HII regions with undetectable or weak temperature-sensitive line ratios Pilyugin (2001). The definition of moderately high metallicity is adopted from Pilyugin (2001) and refers to the abundance interval $8<$ $12+\log \mathrm{O} / \mathrm{H}<8.5$ where the relation $\mathrm{O} / \mathrm{H}=F\left(R_{23}\right)$ (Pagel et al. 1979) degenerates. As suggested by Pilyugin (2001), the positions of HII regions in the $P-R_{3}$ diagram are related to their oxygen abundances. Since both PRGs are in the region where moderately high-metallicity HII regions are located, we can use Eq. (2) above to estimate the oxygen abundances for both UGC 7576 and UGC 9796.

We estimated the mean oxygen abundance parameter, $R_{23}$, by summing the fluxes of the nebular emission lines at different regions along the polar structures (see Table 2). The average values of oxygen abundance obtained for UGC 7576 and UGC 9796 are $12+\log (\mathrm{O} / \mathrm{H})_{P}=8.5 \pm 0.5$ and $12+\log (\mathrm{O} / \mathrm{H})_{P}=7.7 \pm 1$, respectively (see Table 3$)$. Moreover, for UGC 7576 it has been possible to measure the oxygen abundance $12+\log (\mathrm{O} / \mathrm{H})_{P}$ at different distances from the center of the galaxy, and these values are shown in Fig. 7.

\section{Metallicity and SFR estimates}

We derived the oxygen abundance $12+\log (\mathrm{O} / \mathrm{H})$ for UGC 7576 and UGC 9796 by using the empirical P-method (Pilyugin 2001). We found $12+\log (\mathrm{O} / \mathrm{H})=8.5 \pm 0.5$ for UGC 7576 and $12+\log (\mathrm{O} / \mathrm{H})=7.7 \pm 1$ for UGC 9796. The metallicities corresponding to each value of oxygen abundances given before were estimated. We adopted $12+\log (\mathrm{O} / \mathrm{H})_{\odot}=8.83 \pm 0.20=A_{\odot}$ and $Z_{\odot}=0.02$ (Grevesse \& Sauval 1998). Given that $Z \approx K Z_{\odot}$, $K_{\mathrm{UGC}} 7576=10^{\left[A_{\mathrm{UGC}} 7576-A_{\odot}\right]}$ and $K_{\mathrm{UGC}} 9796=10^{\left[A_{\mathrm{UGC}} 9796-A_{\odot}\right]}$, we obtain metallicities for the HII regions of the polar disk of $Z \simeq 0.008$ in UGC 7576 and $Z \simeq 0.002$ in UGC 9796, which correspond to $Z \simeq(0.40 \pm 0.01) Z_{\odot}$ and $Z \simeq(0.100 \pm 0.005) Z_{\odot}$ respectively, in good agreement with the values obtained by (Radtke et al. 2003, see Table 3). 
Table 2. Observed and de-reddened emission line fluxes relative to $\mathrm{H}_{\beta}$.

\begin{tabular}{lccc}
\hline \hline Line & $\lambda(\AA)$ & Observed flux relative to $\mathrm{H}_{\beta}$ & De-reddened flux relative to $\mathrm{H}_{\beta}$ \\
\hline UGC 9796 & & & \\
{$[\mathrm{OII}]$} & 3727 & 8.9 & 6.8 \\
{$[\mathrm{OIII}]$} & 4959 & 5.0 & 5.1 \\
{$[\mathrm{OIII}]$} & 5007 & 8.7 & 9.0 \\
$\mathrm{H}_{\gamma}$ & 4340 & 0.9 & 0.8 \\
$\mathrm{H}_{\alpha}$ & 6563 & 2.2 & 2.8 \\
\hline $\mathrm{UGC} 7576$ & & & \\
{$[\mathrm{OII}]$} & 3727 & 2.5 & 2.8 \\
{$[\mathrm{OIII}]$} & 4959 & 1.5 & 1.51 \\
{$[\mathrm{OIII}]$} & 5007 & 1.7 & 1.71 \\
$\mathrm{H}_{\gamma}$ & 4340 & 1.1 & 1.15 \\
$\mathrm{H}_{\alpha}$ & 6563 & 3.2 & 2.9 \\
\hline
\end{tabular}

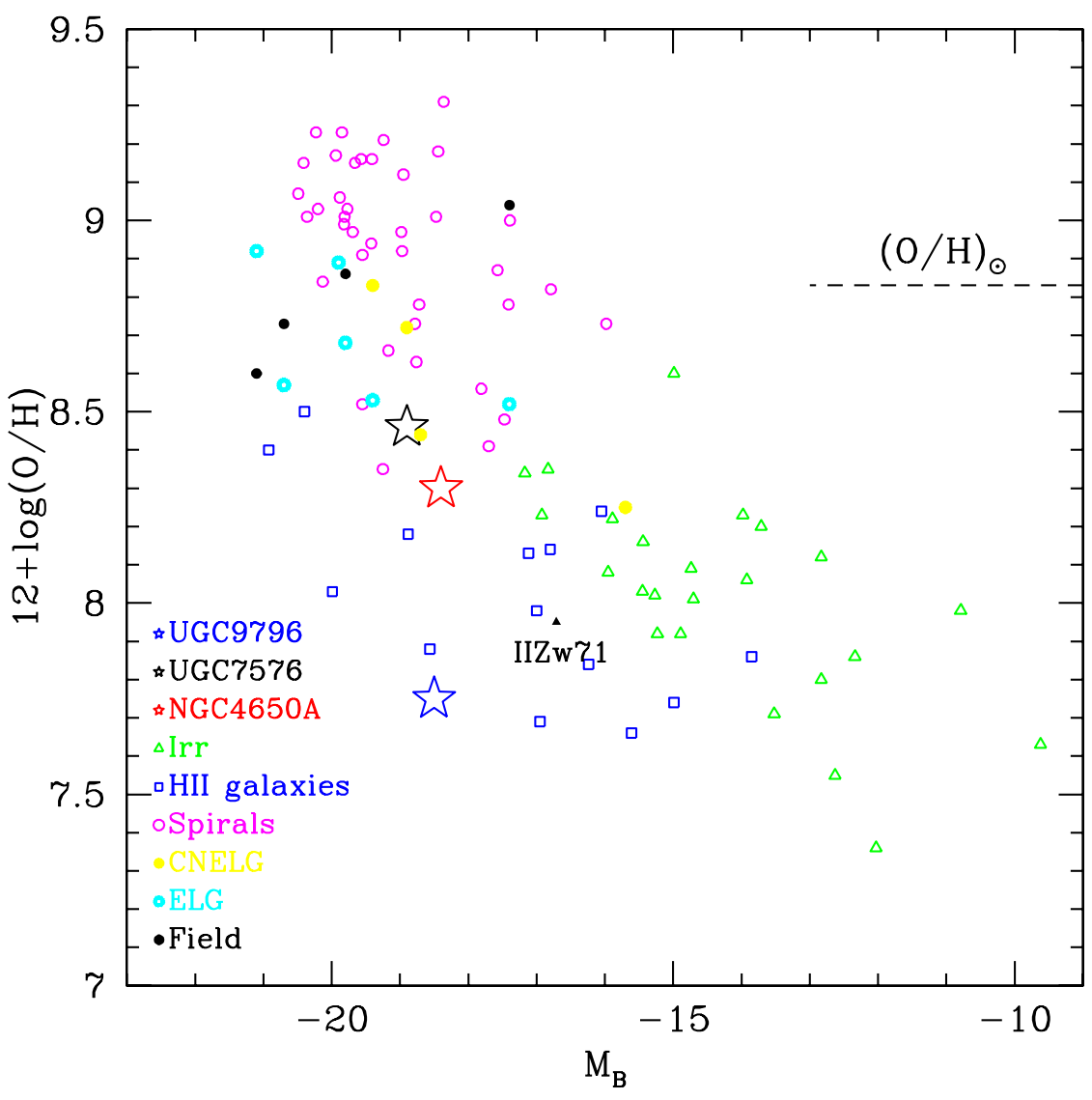

Fig. 6. Oxygen abundance vs. absolute blue magnitude for Compact Narrow Emission-Line Galaxies (CNELGs, yellow filled circles), starforming Emission Line Galaxies (ELGs, cyan open circles), four field galaxies with emission lines (filled black circles), nearby dwarf irregulars (open triangles), local spiral galaxies (open circles), local HII galaxies (open squares), NGC 4650A (red star) (Spavone et al. 2010), the polar disk galaxy IIZw71 (PerezMontero et al. 2009), UGC 7576 (black star), and UGC 9796 (blue star) (this work). The dashed line indicates the solar oxygen abundance.

Table 3. Oxygen abundances and metallicities of UGC 7576 and UGC 9796 compared to those obtained for NGC 4650A.

\begin{tabular}{lccc}
\hline \hline Parameter & UGC 7576 & UGC 9796 & NGC 4650A \\
\hline $12+\log (\mathrm{O} / \mathrm{H})$ & $8.5 \pm 0.5$ & $7.7 \pm 1$ & $8.2 \pm 0.2^{a}$ \\
$Z / Z_{\odot}$ & $0.4 \pm 0.01$ & $0.1 \pm 0.005$ & $0.2 \pm 0.002^{a}$ \\
\hline
\end{tabular}

References. ${ }^{(a)}$ Spavone et al. (2010).

\subsection{Metallicity-luminosity relation}

The mean values for the oxygen abundance along the polar structure of UGC 7576 and UGC 9796, derived by the empirical method (see Sect. 4), are compared with those for a sample of late-type disk galaxies by Kobulnicky \& Zaritsky (1999) as a function of the total luminosity. To this aim, we converted the absolute blue magnitude for the objects in the sample of Kobulnicky \& Zaritsky (1999) by using $H_{0}=75 \mathrm{~km} \mathrm{~s}^{-1} \mathrm{Mpc}^{-1}$. Results are shown in Fig. 6.

We found that UGC 7576 is located in the region where spiral galaxies are found, and in contrast to its high luminosity, it has metallicity lower than spiral galaxy disks of the same total luminosity, and it is consistent with what is observed for NGC 4650A.

UGC 9796 instead is even more metal-poor than UGC 7576 and NGC 4650A, in fact it is located in the region where HII and irregular galaxies are also found, characterized by lower luminosities and metallicities than for the spiral galaxies.

We also compared our new results with those obtained by Pérez-Montero et al. (2009) for IIZw71, a blue compact dwarf 


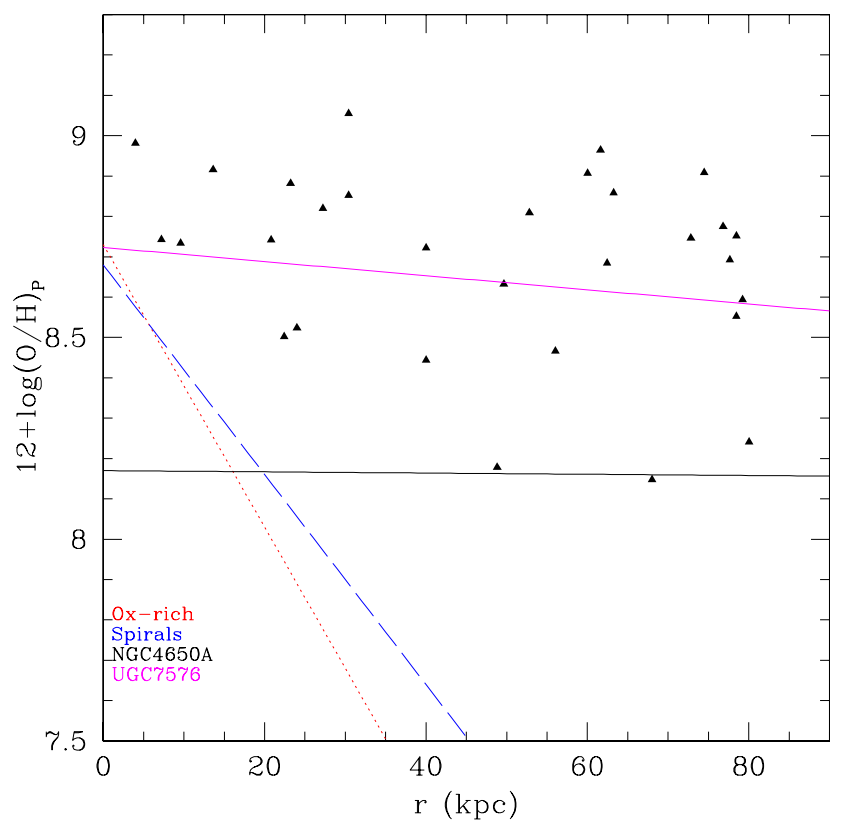

Fig. 7. Oxygen abundance of UGC 7576 derived with P-method, proposed by Pilyugin (2001), versus radius. The superimposed lines are the linear best fit derived by Pilyugin et al. (2006); the red line represents the best fit to the abundance of oxygen-rich spirals, while the blue line is those related to ordinary spirals. The black line is the best fit obtained for NGC 4650A, while the magenta line is the best fit obtained for UGC 7576 .

galaxy also cataloged as a probable polar ring. Consistent with its low luminosity, the metallicity of the brightest knots in the ring of IIZw71 is lower than in UGC 7576, but it is slightly higher than those of UGC 9796. Taking the total magnitude into account, such values are somewhat lower than expected by the metallicity-luminosity relation.

\subsection{Star formation rate}

The $\mathrm{H}_{\alpha}$ emission is detected in both systems with adequate signal-to-noise, and from the measured integrated flux we can derive the star formation rate in the polar ring structure. We derived the SFR for the polar structures of UGC 7576 and UGC 9796, from the $\mathrm{H}_{\alpha}$ luminosity using the expression given by Kennicutt $(1998) S F R=7.9 \times 10^{-42} \times L\left(\mathrm{H}_{\alpha}\right)$. We find that it is almost constant along the disks of both galaxies, within a large scatter in the individual values. From the average values of $L\left(\mathrm{H}_{\alpha}\right) \simeq 2.36 \times 10^{36} \mathrm{erg} / \mathrm{s}$ for UGC 7576 and $L\left(\mathrm{H}_{\alpha}\right) \simeq 5.33 \times 10^{35} \mathrm{erg} / \mathrm{s}$ for UGC 9796 , we have obtained an average $S F R \sim 1.9 \times 10^{-5} M_{\odot} / \mathrm{yr}$ and $S F R \sim 4.2 \times 10^{-6} M_{\odot} / \mathrm{yr}$, respectively. These values are significantly lower than those obtained for NGC 4650A (Spavone et al. 2010), which is SFR $0.06 M_{\odot} / \mathrm{yr}$, and for IIZw71 (Pérez-Montero et al. 2009), which is $S F R \sim 0.035 M_{\odot} / \mathrm{yr}$.

Considering that the polar structure in both PRGs is very young, because the last burst of star formation occurred less than 1 Gyr ago (Reshetnikov \& Combes 1994), we checked if the present SFR and even 2 and 3 times higher (i.e. $S F R=$ $3.8 \times 10^{-5} M_{\odot} / \mathrm{yr}, S F R=5.7 \times 10^{-5} M_{\odot} / \mathrm{yr}$ and $S F R=$ $\left.8.4 \times 10^{-6} M_{\odot} / \mathrm{yr}, S F R=1.26 \times 10^{-5} M_{\odot} / \mathrm{yr}\right)$ can give the inferred metallicities of $Z=0.4 Z_{\odot}$ (for UGC 7576) and $Z=0.1 Z_{\odot}$ (for
UGC 9796) and looked at how strongly they could increase the metallicity with time.

Given the presence of star-forming regions in the polar disk of NGC 4650A, Spavone et al. (2010) used a constant SFR law for this object. For UGC 7576 and UGC 9796, the redder $B-V$ color and the lower $M_{\mathrm{HI}} / L_{B}$ suggest a longer time after the last burst of star formation in these objects. Furthermore, the difference in the detected $\mathrm{H}_{\alpha}$ fluxes, hence of the SFR of these galaxies, is consistent with the above prediction (see Table 1). Given these observational properties and the absence of star-forming clumps, we adopted an exponentially declining SFR that started several Gyrs ago: this is typically used for late-type galaxies because it gives, on average, even older stars than a linear decay. The expression is $\left.\operatorname{SFR}(t)=M_{\star} \tau^{-1} \exp \left[-\left(t_{0}-t\right) / \tau\right)\right]$, where we assume $t_{0}=8 \mathrm{Gyr}$, because in $\Lambda \mathrm{CDM}$ models such cold accretion is more unlikely at low redshifts, $\tau=2 \mathrm{Gyr}$ is the decay timescale and $t$ the lookback time (Bruzual \& Charlot 2003).

By using the mass-metallicity relation derived by Tremonti et al. (2004), where $12+\log (\mathrm{O} / \mathrm{H})=-1.492+1.847 \log \left(M_{\star}\right)-$ $0.08026\left(\log M_{\star}\right)^{2}$, we found that $0.5 Z_{\odot} \leq Z \leq 1 Z_{\odot}$ for UGC 7576 and $0.05 Z_{\odot} \leq Z \leq 0.45 Z_{\odot}$ for UGC 9796. This shows that the present SFR for the polar structures is able to increase the metallicity of about $0.15 Z_{\odot}$ for UGC 7576 and $0.05 Z_{\odot}$ for UGC 9796 , after $1 \mathrm{Gyr}$. We also derived the expected metallicities by using different values for the free parameters $\tau$ (the decay timescale) and $t_{0}$, in order to check how these parameters can change the metallicity range, and found that the obtained metallicities are comparable, or even lower, than those reported above, with differences of about $4 \%$.

For UGC 7576 the derived values for $Z$ are higher than $Z=0.40 \pm 0.01 Z_{\odot}$ found by using the element abundances (see Sect. 4); for UGC 9796, instead, we found that the metallicity of $Z=0.100 \pm 0.005 Z_{\odot}$, estimated by using the element abundances, falls near the lower limit of the range of expected metallicities. The implications of these results will be discussed in detail in Sect. 6.

\subsection{Metallicity gradient along the polar structure of UGC 7576}

The oxygen abundance in the polar ring of UGC 7576 as a function of the radius derived by empirical methods is shown in Fig. 7, where the plot shows that the metallicity remains almost constant along the projected major axis of the polar ring. It is only slightly declining, unlike what it is observed for spiral galaxies, where there is a very steep gradient in metallicity. The absence of a metallicity gradient is a typical behavior found in LSB galaxies (de Blok \& van der Hulst 1998), as in the polar disk galaxy studied by Brooks et al. (2009) and in NGC 4650A (Spavone et al. 2010). This suggests that, as already pointed out for NGC 4650A (Spavone et al. 2010), the star formation and the metal enrichment in the polar structure is not influenced by the stellar evolution of the central spheroid and that the polar ring was formed later. As also suggested by Rupke et al. (2010), numerical simulations predict that the absence of metallicity gradient is accounted for by the inflow of low metallicity gas from the outskirts.

In contrast, ordinary and oxygen-rich spiral galaxies, where no primordial gas is accreted from outside, show a decreasing abundance with increasing radii (see Fig. 7 and Pilyugin et al. 2006). These observed features in spiral disks are explained well by the infall models of galaxy formation that predict that these systems build up through the accretion of onsite gas. This gas becomes more metal rich while it flows towards the galaxy center 
Table 4. Discriminating parameters between different formation scenarios.

\begin{tabular}{|c|c|c|c|c|c|c|c|c|c|}
\hline PRG & $\begin{array}{l}M_{b}^{\mathrm{HG}} \\
\left(M_{\odot}\right)\end{array}$ & $\begin{array}{l}M_{b}^{\mathrm{PD}} \\
\left(M_{\odot}\right) \\
\end{array}$ & $\begin{array}{c}V_{\mathrm{eq}} \\
\left(\mathrm{km} \mathrm{s}^{-1}\right)\end{array}$ & $\overline{V_{\text {eq }} / V_{\mathrm{PD}}}$ & $\begin{array}{c}\sigma_{0} \\
\left(\mathrm{~km} \mathrm{~s}^{-1}\right)\end{array}$ & $M_{b}^{\mathrm{HG}} / M_{b}^{\mathrm{PD}}$ & $v / \sigma$ & $\begin{array}{l}Z_{\text {est }} \\
\left(Z_{\odot}\right)\end{array}$ & $\begin{array}{l}Z_{\exp } \\
\left(Z_{\odot}\right) \\
\end{array}$ \\
\hline UGC 7576 & $7.86 \times 10^{9}$ & $2.88 \times 10^{9}$ & 212 & 0.96 & 116 & 2.73 & 1.8 & $(0.40 \pm 0.01)$ & $(0.5 \div 1)$ \\
\hline UGC 9796 & $1.0 \times 10^{10}$ & $3.05 \times 10^{9}$ & 157 & 1.08 & 73 & 3.28 & 2.15 & $(0.100 \pm 0.005)$ & $(0.05 \div 0.45)$ \\
\hline NGC 4650A & $5 \times 10^{9}$ & $12 \times 10^{9}$ & 90 & 0.75 & 70 & 0.42 & 1.28 & $(0.200 \pm 0.002)$ & $(1.02 \div 1.4)$ \\
\hline
\end{tabular}

(Matteucci \& Francois 1989; Boissier \& Prantzos 1999), and this process generates the observed gradients.

\section{Formation history for UGC 7576 and UGC 9796: discussion and conclusions}

Galaxies with a polar ring/disk play a special role in studies of the physical processes at work during galaxy interactions and merging. To account both for the featureless morphology of the central spheroidal galaxy and for the more complex structure of the polar ring/disk, three main formation processes have been proposed so far: i) a major dissipative merger; ii) tidal accretion of material (gas and/or stars) from outside; and iii) cold accretion of pristine gas along a filament.

In the merging scenario, the PRG results from a "polar" merger of two disk galaxies with unequal mass, (Bekki 1997, 1998; Bournaud et al. 2005). In the accretion scenario, the polar ring/disk may form by a) the disruption of a dwarf companion galaxy orbitating around an early-type system or by b) the tidal accretion of gas stripping from a disk galaxy outskirts, captured by an early-type galaxy on a parabolic encounter (Reshetnikov \& Sotnikova 1997; Bournaud \& Combes 2003; Hancock et al. 2009). Both major merger and accretion scenarios are able to account for many observed PRGs morphologies and kinematics, such as the existence of wide and narrow rings, helical rings, and double rings (Whitmore et al. 1990).

The cold accretion scenario has been proposed very recently for the formation of wide disk-like polar rings (see Sect. 1). A long-lived polar structure may form through cold gas accretion along a filament, extended for $\sim 1 \mathrm{Mpc}$, into the virialized dark matter halo (Macciò et al. 2006). In this formation scenario, there are no limits to the mass of the accreted material, so a very massive polar disk may develop around either a stellar disk or a spheroid.

As suggested by the previous studies of PRGs (Spavone et al. 2010; Iodice et al. 2006), the critical physical parameters that can tell the differences between the three formation scenarios are 1) the total baryonic mass (stars plus gas) observed in the polar structure with respect to the central spheroid; 2) the kinematics along both the equatorial and meridian planes; 3 ) the metallicity and SFR in the polar structure.

In the tidal accretion scenario, the total amount of accreted gas by the early-type object is about $10 \%$ of the gas in the disk donor galaxy, i.e. up to $10^{9} M_{\odot}$. In the case of UGC 7576 and UGC 9796, the polar structure is characterized by a high baryonic mass (see Table 4), comparable to the total luminous mass in the central spheroid. Similar to the case of NGC 4650A (Spavone et al. 2010), the high HI mass excludes formation of the polar structures through the tidal accretion of gas and stars by an external donor galaxy (Bournaud \& Combes 2003).

In the merging scenario, the morphology and kinematics of the merger remnants depend on the merging initial orbital parameters and the initial mass ratio of the two galaxies (Bournaud et al. 2005). For UGC 7576 and UGC 9796, this scenario is ruled out because, according to simulations (e.g. Bournaud et al. 2005), a high mass ratio of the two merging galaxies is required to form a massive and extended polar disk as observed in both the PRGs. This would convert the intruder into an ellipticallike, not rotationally supported, stellar system. Since this contrasts with the high maximum rotation velocities observed (see Reshetnikov \& Combes 1994; and Table 4) in UGC 7576 $\left(\sim 212 \mathrm{~km} \mathrm{~s}^{-1}\right)$ and UGC $9796\left(\sim 157 \mathrm{~km} \mathrm{~s}^{-1}\right)$, the merging scenario is ruled out for both galaxies.

As already mentioned, if the polar structure around both an elliptical and disk galaxy forms by the cold accretion of gas from filaments there is no limit to the accreted mass. Moreover, due to the inflow of pristine gas, the metallicity is lower than that observed in galaxies of a comparable total luminosity, and the value derived by the present SFR is higher than those directly measured by the chemical abundances. Spavone et al. (2010) find that such predictions are consistent with observations in the polar disk galaxy NGC 4650A, leading to the conclusion that the cold accretion of gas by cosmic web filaments is the most realistic formation scenario for this object.

By studying the chemical abundances in the polar structure of UGC 7576 and UGC 9796, we aim at testing the cold accretion scenario for these objects. The main results are as follows (see also Sect. 5):

1. both PRGs have on average lower metallicity with respect to that of same-luminosity spiral disks, in particular, for UGC $7576 Z=0.4 Z_{\odot}$ and for UGC $9796 Z=0.1 Z_{\odot}$;

2. the metallicities expected for the present SFR at three different epochs are higher than those measured from the element abundances, and they vary in the range $0.5 Z_{\odot} \leq Z \leq 1 Z_{\odot}$ for UGC 7576 and $0.05 Z_{\odot} \leq Z \leq 0.45 Z_{\odot}$ for UGC 9796;

3. for UGC 7576, the metallicity remains almost constant along the polar structure.

In the following, we address how these results can be reconciled with the theoretical predictions for the cold accretion process and discuss how the SFR and metallicity derived in this work, together with the other two key parameters, baryonic mass and kinematics, available from previous studies of these objects, may help to distinguish among the three formation scenarios, in unambiguous ways as done for NGC 4650A. All these quantities for both the PRGs studied in this work and NGC 4650A, are summarized in Table 4.

The cold accretion mechanism for disk formation predicts rather low metallicity $\left(Z=0.1 Z_{\odot}\right)$ (Dekel \& Birnboim 2006; Ocvirk et al. 2008; Agertz et al. 2009). This value refers to the time just after the accretion of a misaligned material, so it can be considered as an initial value for $Z$ before the subsequent enrichment. How can this be reconciled with the observed metallicity for UGC 7576 and UGC 9796?

We estimated that the present SFR for the polar structure is $S F R=1.9 \times 10^{-5} M_{\odot} \mathrm{yr}^{-1}$ for UGC 7576 and $S F R=4.2 \times$ $10^{-6} M_{\odot} \mathrm{yr}^{-1}$ for UGC 9796 . These values are able to increase the metallicity of about $0.15 Z_{\odot}$ for UGC 7576 and $0.05 Z_{\odot}$ for 
UGC 9796 after 1Gyr (see Sect. 5). Considering that the polar structure is very young, less than 1 Gyr (Reshetnikov et al. 1994), an initial value of $Z=0.1 Z_{\odot}$ at the time of polar structure formation could be consistent with today's observed metallicity. Therefore, the cold accretion of gas can be a possible formation scenario for both PRGs, UGC 7576 and UGC 9796. In the case of UGC 7576, one more hint of the cold accretion scenario comes from the fact that the metallicity expected by the present SFR turns to be higher than those directly measured by the chemical abundances. As already suggested for NGC 4650A (Spavone et al. 2010), a possible explanation for this observed feature could be the infall of pristine gas (Finlator et al. 2007; Ellison et al. 2008).

Moreover, for UGC 7576, the lack of an abundance gradient along the polar structure is consistent with the prediction of recent numerical simulations by Rupke et al. (2010) and Kewley \& Ellison (2008), who observe flatter metallicity gradients than those observed in typical spiral galaxies, owing to the radial inflow of low-metallicity gas from outside. For UGC 9796, instead, the metallicity derived by the chemical abundances falls near the lower limit of the expected metallicity range, and for this reason we cannot definitely rule out other possible formation scenarios.

The high baryonic (gas+stars) masses, the large extensions of the polar structure, and the low metallicity observed in these PRGs are not accounted for in the formation of polar rings through the disruption of a dwarf galaxy, which are characterized by higher metallicities $\left(1 / 9 Z_{\odot} \div 1 / 3 Z_{\odot}\right)$ and lower HI masses ( $\sim 10^{7} M_{\odot}$; Galametz et al. 2009; and Bekki 2008).

Differently, the tidal accretion scenario, in which gas is stripped from a gas-rich donor in a particular orbital configuration (Bournaud \& Combes 2003), is able to produce wide polar rings and/or disks both around a disk or an elliptical galaxy. In order to consider the accretion hypothesis, we studied the field around these galaxies to see if there are any objects as possible donor galaxy candidates: inside a radius of about five times the diameter of both PRGs, as suggested by Brocca et al. (1997), we find that UGC 7576 has no close companions, while UGC 9796, that is in a small group, has five companions (Cox et al. 2006). Therefore, while the tidal accretion scenario is also ruled out for UGC 7576, it needs to be envisioned in the case of UGC 9796.

The VLA images show that UGC 9796 and the close companion galaxy MCG+07-31-049 (labeled as 1 in Fig. 8) could be in the orbital configuration needed to form a polar structure through an accretion event (Bournaud \& Combes 2003). In fact, the HI gas in UGC 9796, which is all associated with the polar structure, has the outer contours warped away from the poles, and in the SE regions, it extends towards MCG+0731-049, which has an HI distribution extending in a direction perpendicular to that of UGC 9796.

In the tidal accretion process, the accreted gas only comes from the outer and more metal-poor regions of the donor galaxy. All the companion galaxies of UGC 9796 have an amount of HI gas $\left(\sim 10^{9} M_{\odot}\right)$ comparable to those of this PRG (Cox et al. 2006), but according to Bresolin et al. (2009), the metallicity of the outer regions of bright spiral galaxies is $0.2 Z_{\odot} \leq Z \leq 1.1 Z_{\odot}$ and the observed value for UGC 9796, $Z=0.100 \pm 0.005 Z_{\odot}$, is below the lower limit.

Given all the evidence shown above, we can give the following conclusions for this work. The cold accretion of gas by cosmic web filaments could well account for the low metallicity, the lack of gradient, and the high HI content in UGC 7576. Moreover, the general underdensity of the environment where

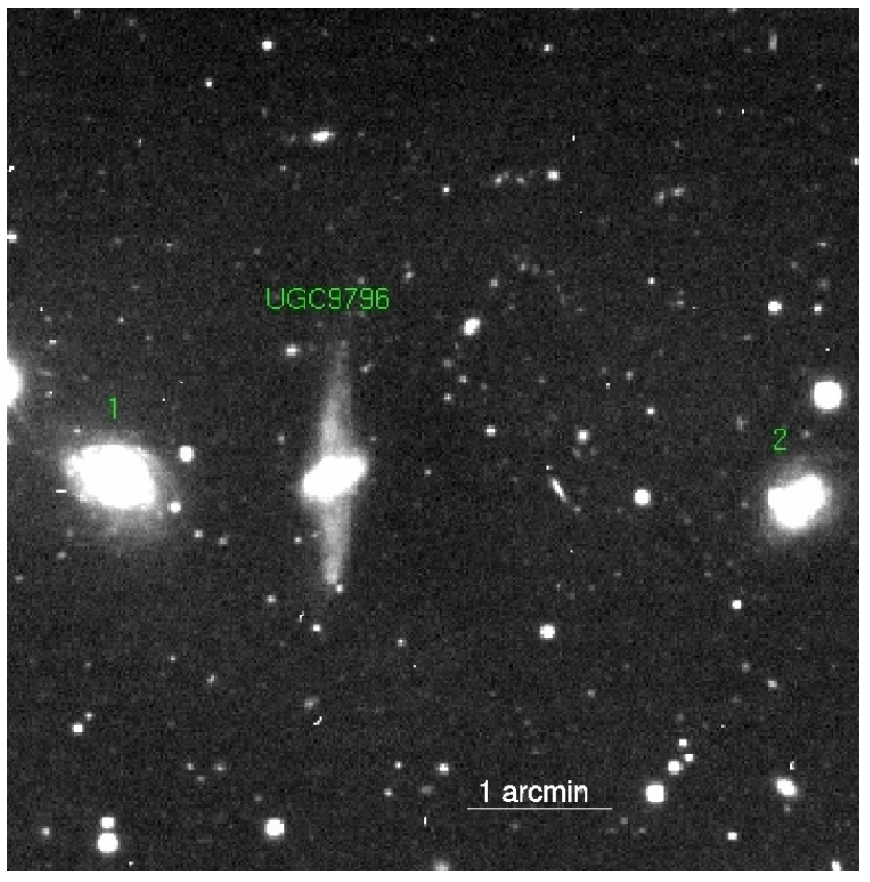

Fig. 8. Optical image of UGC 9796 and its two nearest companion galaxies, MCG +07-31-049 (number 1) and CGPG 1514.2+4320 (number 2).

this galaxy is can be consistent with the cold flow accretion of gas as the possible formation mechanism for this object.

For UGC 9796 instead, the scenario is slightly more complex. In fact, the low estimated metallicity, even lower than observed in the outskirts of spiral galaxies is consistent with the formation of disks through cold accretion mechanisms (Dekel \& Birnboim 2006; Ocvirk et al. 2008; and Agertz et al. 2009). On the other hand, the tidal accretion scenario cannot be ruled out, given that the galaxy MCG +07-31-049, with its high amount of $\mathrm{HI}$ and its orbital configuration, could be a good candidate donor for UGC 9796.

Acknowledgements. The authors wish to thank the anonymous referee for the detailed and constructive report, and Frederic Bournaud for many useful discussions and suggestions. M.S. and E.I. wish to thank Massimo Capaccioli for his useful suggestions, which allowed us to improve this paper. This work is based on observations made with Telescopio Nazionale Galileo (TNG) under program ID A21TAC-54.

\section{References}

Agertz, O., Teyssier, R., \& Moore, B. 2009, MNRAS, 397, L64 Arnaboldi, M., Oosterloo, T., Combes, F., Freeman, K. C., \& Koribalski, B. 1997, AJ, 113, 585

Bekki, K. 1997, ApJ, 490, L37

Bekki, K. 1998, ApJ, 499, 635

Bekki, K. 2008, ApJ, 680, L29

Boissier, S., \& Prantzos, N. 1999, MNRAS, 307, 857

Bournaud, F., \& Combes, F. 2003, A\&A, 401, 817

Bournaud, F., \& Elmegreen, B. G. 2009, ApJ, 694, L158

Bournaud, F., Jog, C. J., \& Combes, F. 2005, A\&A, 437, 69

Bournaud, F., Jog, C. J., \& Combes, F. 2007, A\&A, 476, 1179

Bresolin, F., Ryan-Weber, E., Kennicutt, R. C., \& Goddard, Q. 2009, ApJ, 695, 580

Brocca, C., Bettoni, D., \& Galletta, G. 1997, A\&A, 326, 907

Brook, C. B., Governato, F., Quinn, T., et al. 2008, ApJ, 689, 678

Brooks, A. M., Governato, F., Quinn, T., Brook, C. B., \& Wadsley, J. 2009, ApJ, 694, 396

Brosch, N., Kniazev, A. Y., Moiseev, A., \& Pustilnik, S. A. 2010, MNRAS, 401, 2067

Bruzual, G., \& Charlot, S. 2003, MNRAS, 344, 1000 
Cardelli, J. A., Clayton, G. C., \& Mathis, J. S. 1989, ApJ, 345, 245

Cole, S., Lacey, C. G., Baugh, C. M., \& Frenk, C. S. 2000, MNRAS, 319, 168

Conselice, C. J., Bershady, M. A., Dickinson, M., \& Papovich, C. 2003, AJ, 126, 1183

Cox, A. L., Sparke, L. S., \& van Moorsel, G. 2006, AJ, 131, 828

de Blok, W. J. G., \& van der Hulst, J. M. 1998, A\&A, 335, 421

De Lucia, G., Springel, V., White, S. D. M., Croton, D., \& Kauffmann, G. 2006, MNRAS, 366, 499

Dekel, A., \& Birnboim, Y. 2006, MNRAS, 368, 2

Dekel, A., \& Birnboim, Y. 2008, MNRAS, 383, 119

Dekel, A., Sari, R., \& Ceverino, D. 2009a, ApJ, 703, 785

Dekel, A., Birnboim, Y., Engel, G., et al. 2009b, Nature, 457, 451

Díaz, A. I., \& Pérez-Montero, E. 2000, MNRAS, 312, 130

Ellison, S. L., Patton, D. R., Simard, L., \& McConnachie, A. W. 2008, ApJ, 672, L107

Elmegreen, D. M., Elmegreen, B. G., Ravindranath, S., \& Coe, D. A. 2007, ApJ, 658,763

Finlator, K., Davé, R., \& Oppenheimer, B. D. 2007, MNRAS, 376, 1861

Galametz, M., Madden, S., Galliano, F., et al. 2009, A\&A, 508, 645

Gallagher, J. S., Sparke, L. S., Matthews, L. D., et al. 2002, ApJ, 568, 199

Genel, S., Genzel, R., Bouché, N., et al. 2008, ApJ, 688, 789

Grevesse, N., \& Sauval, A. J. 1998, SSR, 85, 161

Hancock, M., Smith, B. J., Struck, C., Giroux, M. L., \& Hurlock, S. 2009, AJ, 137,4643

Iodice, E., Arnaboldi, M., De Lucia, G., et al. 2002a, AJ, 123, 195

Iodice, E., Arnaboldi, M., Sparke, L. S., Gallagher, J. S., \& Freeman, K. C. 2002b, A\&A, 391, 103

Iodice, E., Arnaboldi, M., Saglia, R. P., et al. 2006, ApJ, 643, 200

Katz, N., \& White, S. D. M. 1993, ApJ, 412, 455

Katz, N., Quinn, T., Bertschinger, E., \& Gelb, J. M. 1994, MNRAS, 270, L71

Kennicutt, R. C., Jr. 1998, ARA\&A, 36, 189

Kennicutt, R. C., Jr., Roettiger, K. A., Keel, W. C., van der Hulst, J. M., \& Hummel, E. 1987, AJ, 93, 1011
Keres, D. 2008, 37th COSPAR Scientific Assembly, 37, 1496

Kereš, D., Katz, N., Weinberg, D. H., \& Davé, R. 2005, MNRAS, 363, 2

Kewley, L. J., \& Ellison, S. L. 2008, ApJ, 681, 1183

Kobulnicky, H. A., \& Zaritsky, D. 1999, ApJ, 511, 118

Macciò, A. V., Moore, B., \& Stadel, J. 2006, ApJ, 636, L25

Masana, E., Jordi, C., Maitzen, H. M., \& Torra, J. 1998, A\&A, 128, 265

Matteucci, F., \& Francois, P. 1989, MNRAS, 239, 885

Naab, T., Johansson, P. H., Ostriker, J. P., \& Efstathiou, G. 2007, ApJ, 658, 710

Ocvirk, P., Pichon, C., \& Teyssier, R. 2008, MNRAS, 390, 1326

Pagel, B. E. J., Edmunds, M. G., Blackwell, D. E., Chun, M. S., \& Smith, G. 1979, MNRAS, 189, 95

Pérez-Montero, E., \& Díaz, A. I. 2003, MNRAS, 346, 105

Pérez-Montero, E., García-Benito, R., Díaz, A. I., Pérez, E., \& Kehrig, C. 2009, A\&A, 497, 53

Pilyugin, L. S. 2001, A\&A, 369, 594

Pilyugin, L. S., Thuan, T. X., \& Vílchez, J. M. 2006, MNRAS, 367, 1139

Radtke, I. R., Eskridge, P. B., \& Pogge, R. W. 2003, BAAS, 35, 757

Reshetnikov, V. P., \& Combes, F. 1994, A\&A, 291, 57

Reshetnikov, V., \& Sotnikova, N. 1997, A\&A, 325, 933

Reshetnikov, V. P., Hagen-Thorn, V. A., \& Yakovleva, V. A. 1994, A\&A, 290 693

Robertson, B. E., \& Bullock, J. S. 2008, ApJ, 685, L27

Roškar, R., Debattista, V. P., Brooks, A. M., et al. 2010, MNRAS, 408, 783

Rupke, D. S. N., Kewley, L. J., \& Barnes, J. E. 2010, ApJ, 710, L156

Schechter, P. L., Sancisi, R., van Woerden, H., \& Lynds, C. R. 1984, MNRAS, 208, 111

Spavone, M., Iodice, E., Arnaboldi, M., et al. 2010, ApJ, 714, 1081

Springel, V., \& Hernquist, L. 2005, ApJ, 622, L9

Swaters, R. A., \& Rubin, V. C. 2003, ApJ, 587, L23

Tremonti, C. A., Heckman, T. M., Kauffmann, G., et al. 2004, ApJ, 613, 898

Whitmore, B. C., Lucas, R. A., McElroy, D. B., et al. 1990, AJ, 100, 1489 